\title{
Aequalitas essendi en la obra De Docta Ignorantia de Nicolás de Cusa
}

\author{
MAURICE AlVARADO CORDERO \\ Pontificio Seminario Mayor San Rafael (Chile) \\ maurice.ac@gmail.com
}

\begin{abstract}
Resumen
Abordamos la cuestión del ser a través de la comprensión que tiene Nicolás de Cusa del Verbo como la Igualdad del ser (Aequalitas essendi) en su obra De Docta Ignorantia. Este concepto está íntimamente relacionado con la Trinidad y la creación. Además veremos como la encarnación de la Igualdad del ser fue necesaria para la misma creación del universo.
\end{abstract}

Palabras clave: ser, igualdad, cristología, creación, encarnación, docta ignorancia, coincidencia de los opuestos.

\section{Aequalitas essendi in Nicholas of Cusa's De Docta Ignorantia}

\begin{abstract}
The question of being is taken into consideration by Nicholas of Cusa's conception of the Word as Equality of being (Aequalitas essendi). This notion is intimately related to the Trinity and to creation itself. As well, we shall see how the incarnation of the Equality of being was necessary for the creation of the entire universe.
\end{abstract}

Key words: being, equality, christology, creation, incarnation, learned ignorance, coincidence of opposites.

Magíster en Teología Dogmática por la Pontificia Universidad Católica de Chile. Sacerdote de la diócesis de Valparaíso (Chile). Profesor de teología en el Pontificio Seminario Mayor San Rafael. Este artículo representa una visión de síntesis de una investigación anterior que fue presentada en la Pontificia Universidad Católica de Chile para optar al grado de Magíster en Teología Dogmática. 


\section{Introducción: el Cusano y el problema del ser}

Las grandes preguntas del ser humano se reducen finalmente a una: ¿ser o no ser? No se trata de la pregunta de la obra de Hamlet de William Shakespeare, sino que es una pregunta aún más antigua de la cual tenemos vestigios ya en el poema de Parménides. La cuestión del ser es recogida a lo largo de toda la filosofía. También es la cuestión detrás de toda religión en el sentido que trata de dar explicación a la existencia del ser, de lo que es. Hoy en día la se resume esta pregunta en las palabras de Martín Heidegger (2001): «¿Por qué es el ente y no más bien la nada?» Esta pregunta supone algún origen del ser. No es monopolio de la religión decir que ese origen está en Dios. También la filosofía a lo largo de milenios ha intentado descubrir este origen de todo, el origen sin origen. En el cristianismo esta pregunta se resuelve principalmente en la revelación divina y esta revelación llega a su plenitud en Jesucristo. Ahora bien, ha sido tradición desde siempre que el cristianismo intente buscar respuestas en que fe y razón concuerden. De este modo en el cristianismo se ha sostenido de sus inicios que Dios, origen de todo, trajo todo ser que no fuera Él mismo a la existencia ex nibilio. La creación de la nada en el cristianismo supone una voluntad divina, y en última instancia se dirá que Dios creó todo de la nada por amor.

En la búsqueda sobre la cuestión del ser tenemos un gran testimonio de esfuerzo por tratar de unificar la fe con la razón en el cardenal alemán del siglo XV, Nicolás de Cusa. El Cusano, como comúnmente se le llama, no sólo intenta armonizar fe y razón, sino que también hace un esfuerzo por recopilar lo ya hecho en esta línea desde Platón hasta sus propios días, tiempo en que se vivía la transición entre la Edad Media y el Renacimiento. Aunque es de influencia principalmente neo-platónica, incluye elementos aristotélicos y tomistas. Pero su esfuerzo no es sólo a nivel racional en el ámbito de lo filosófico-teológico, sino que en su síntesis intenta también asumir una mística basada en el intelecto humano. Su camino filosófico es un intento de unificar las mentalidades cristianas de oriente y occidente de su época, combinar el pensamiento teológico de ambas tradiciones. Aparte de todo esto, una de las cosas que más ha caracterizado al Cusano es su uso de las matemáticas y geometría como camino para comprender y explicar lo divino.

En esto que podríamos llamar hoy en día una teoría unificada el Cusano nos da una gran luz para ir respondiendo a la cuestión del ser. Esta respuesta está en su concepción de Jesucristo como Aequalitas essendi, es decir, la Igualdad del ser. Él es Aequalitas essendi, no sólo si lo consideramos como el Hijo consustancial con el Padre o el Logos existente desde antes de la creación sino considerándolo también como 
el Jesús histórico que vivió en Nazaret. Según el Cusano, el misterio del Verbo encarnado es la respuesta sobre el misterio del ser.

Aunque el pensamiento de Nicolás de Cusa es fundamentalmente cristocéntrico, y es considerado como «pan-Christic ontology» (Albertson, 2006), es escaso el material secundario sobre su cristología en comparación con otros autores. Al parecer la obra Die Christolologie Des Nikolaus Von Kues de Haubst (1956) es el único libro dedicado a la cristología del Cusano. En los últimos años ha aumentado el interés en esta área y se ha destacado su potencial para la teología actual (Balthasar, 1997; Balthasar 1998; Cordovilla Pérez, 2004; Hubert, 2009). Sin embargo, queda aún mucho por investigar. El presente artículo tiene la intención de aportar algo al creciente interés del Cusano con la reflexión sobre el poco considerado pero importantísimo concepto cristológico de Aequalitas essendi.

Investigaremos el uso de este concepto en la obra De Docta Ignorantia. Veremos cómo éste es uno de los ejes centrales de todo el pensamiento de Nicolás de Cusa y a través de este concepto intentaremos abordar la cuestión del ser. Se escogió investigar la obra De Docta Ignorantia debido a que es la obra central del cardenal y además porque es considerada por varios por tener la reflexión más sistemática de su cristología (Tilliette, 1993; Balthasar, 1998; Euler, 2000).

\section{Algunos conceptos claves del Cusano en De Docta Ignorantia}

Antes de entrar a ver el mismo concepto cristológico de aequalitas es necesario revisar algunos otros conceptos claves que rigen el pensamiento del Cusano. Por lo general es conocido por sus aportes filosóficos y gnoseológicos, en particular por su pensamiento de la docta ignorancia y la coincidencia de los opuestos. Dentro de este pensamiento se destaca su idea de lo infinito expresado con los medios matemáticos y geométricos de su tiempo. Es un pensamiento altamente abstracto, que invita a, dentro de una cierta logicidad, romper los esquemas mentales de cantidad, espacio y tiempo. La base de su docta ignorancia es que el conocimiento es conjetural, nunca conocemos algo en toda su verdad. Es aquí que el verdadero saber reconoce que no conoce todo ni algo en su totalidad. Esto es cierto sobre todo cuando el hombre intenta conocer a Dios, el Máximo Absoluto (como lo denomina el Cusano), donde por ser infinito, el mínimo y el máximo, acto y potencia, ser y no ser, coinciden. De este modo, Nicolás de Cusa nos invita a ir más allá del principio de no-contradicción al llegar a pensar que en Dios los opuestos o 
contradictorios coinciden, y que en realidad aún así, Dios está más allá de esa coincidencia.

Una de sus principales ideas es el principio de la noproporcionalidad, es decir que no existe proporcionalidad entre lo finito y lo infinito (Cusa, 1932: 27) ${ }^{1}$. Esta es la base del método de su docta ignorancia. Según él, el conocimiento se da por medio de la comparación entre algo conocido y algo desconocido, y se forma una cierta proporción entre lo primero y lo segundo. Ahora bien, cuando se trata del conocimiento sobre Dios, éste se hace imposible debido a que no hay proporción entre lo creado-finito y Dios-infinito.

Por otra parte, a pesar de la proporcionalidad que el intelecto humano puede observar entre un ser creado y otro, nunca tendrá un conocimiento exacto de las cosas. Esto porque la igualdad perfecta con otro ser no se puede alcanzar sin una duplicidad exactísima del mismo ser, y esto sería imposible porque la única forma que un ser o ente sea exactamente igual al otro sería que fuese ese mismo ser o ente. Entendemos que por principio de identidad, sólo un mismo ser puede ser igual a sí mismo. Por tanto, ni siquiera un ente de razón en el intelecto humano será idéntico al ente real que intenta conocer. Hilando un poco más fino, entendemos que esta igualdad exacta sólo se da en Dios, siendo que la identidad consigo mismo es de orden hipostático, es decir, que el Hijo es la aequalitas del Padre. De modo análogo, las creaturas sólo tienen su igualdad exacta en la mente de Dios, que es el Hijo y que es todo lo que el Padre es porque es su aequalitas.

El gran problema para él será cómo conocer a Dios que es infinito cuando todo lo que tenemos para conocerlo son medios finitos. Es más, cómo es posible que seres finitos provengan de un ser infinito cuando no existe ninguna proporcionalidad entre ellos. Además, si todas las creaturas provienen de un mismo ser, porqué son tan distintas entre sí. Nos enfrentamos al problema del ser junto con los dilemas sobre la alteridad, la finitud e infinitud, de si es posible la analogía entis, y por último sobre el problema de la quididad de los entes.

Nos detendremos un instante en la cuestión sobre la quididad. Para el Cusano éste es un problema fundamental. Como una breve síntesis de lo que hemos estado viendo hasta ahora, dice lo siguiente:

1 Debido a las variadas ediciones de De Docta Ignorantia de Nicolás de Cusa, y para facilitar la búsqueda, en este artículo todas las referencias a la obra apuntan a la famosa edición preparada por Hoffmann y Kliblansky y publicada por la Academia de Heidelburgo en 1932. Con esta numeración se pueden encontrar las citas también en el Cusanus-Portal (http://www.cusanus-portal.de). Sin embargo, todas las traducciones al español de los textos, salvo algunas pequeñas modificaciones, son de la versión de 1984 de La Docta Ignorancia traducida por M. Fuente Benot. 
La quididad de las cosas, por consiguiente, que es la verdad de los entes, es en su puridad inalcanzable, y ha sido investigada por todos los filósofos, pero no ha sido hallada, en cuanto tal, por ninguno. Y cuanto más profundamente doctos seamos en esta ignorancia, tanto más nos acercaremos a la misma verdad (Cusa, 1932: 9).

Mucho más adelante en su vida, en su última obra, De Apice Theoriae, identificará explícitamente el quid de todos los entes con el mismo Hijo de Dios. Pero ya en De Docta Ignorantia tenemos suficientes elementos para ver el Verbo encarnado como la esencia o forma de las cosas.

Muy de la mano de su concepto de infinito que anteriormente mencionamos está su concepción de Dios como maximum:

...llamo máximo (maximum) a aquello, mayor que lo cual nada puede haber. Pero la abundancia conviene a lo uno (unum). Así, con la maximidad coincide la unidad (unitas), que es también entidad (entitas) (Cusa, 1932: 7).

En la cita mencionada vemos algo de influencia del argumento ontológico de San Anselmo: Dios es aquel que es más grande de lo que se puede concebir. Es quizás con la influencia de éste que Nicolás llegará a su teoría de la docta ignorancia. La docta ignorancia sigue por ese mismo camino admitiendo que la existencia máxima de Dios, no es sólo a nivel cognoscitivo sino también real. Aquí el Cusano relaciona el maximum con la unitas. El concepto de unitas se refiere a la divinidad en general, pero en muchas otras ocasiones veremos al Cusano utilizarlo preferentemente para referirse al Padre. Ahora bien, también se puede pensar que maximum se atribuye al Hijo. Se puede entender entonces que maximum y unitas coinciden en el sentido de la unión del Padre y el Hijo. Por eso declara en otra parte que «cuando digo que la unidad es máxima (unitas est maxima), hablo de la trinidad» (Cusa, 1932: 21). Vemos en casos como éste la profunda noción de la perijorésis que maneja el Cusano.

La unitas es considerada también como entitas, lo cual significa que no es mera abstracción, tiene una consistencia real como ente. En algunas ocasiones dirá que Dios es la entidad de todas las cosas, pero no se debe entender en un sentido panteísta sino como origen de toda entidad del mismo modo que decimos que Dios es la bondad misma y que todos los demás seres participan de su bondad. En el concepto de Dios como entitas tenemos la base para distinguirlo de todo otro ente y para el desarrollo de la analogía entis. Pero esto sólo servirá como base para luego con el concepto de Dios como maximum introducirnos por medio de la coincidencia de los opuestos en la docta ignorancia que culmina en la alta 
contemplación de la teología negativa o apofática que no permite ningún tipo de comparación.

\section{La Trinidad como Unitas, Aequalitas y Connexio}

La especulación metafísica del Cusano nos llevará luego a una sugerente concepción trinitaria, llena de dinamismo, solidez teológica y múltiples aplicaciones que el mismo Cusano utilizará a lo largo de su vida.

Entendiendo que en lo central de su pensamiento está la noción neoplatónica de lo Uno intenta demostrar a lo largo del primer libro de De Docta Ignorantia que lo Uno es esencialmente trino. La unidad de la Trinidad es de tal manera que las relaciones trinitarias son interdependientes entre sí de modo que la Trinidad no puede ser sino una, y lo Uno no puede ser sino trino. Para hablar de algún modo sobre la Trinidad y sus relaciones adopta un vocabulario casi matemático que toma de algunos «santos doctores» (Cusa, 1932: 18). Atribuye al Padre el nombre de Unitas; al Hijo, Aequalitas; y al Espíritu Santo, Connexio. Al parecer esta nomenclatura es utilizada por primera vez por Agustín (1845a: 21). Su uso es comentado por el Pseudo-Beda, Thierry de Chartres, Juan de Salisbury, Pedro Lombardo y Tomás de Aquino, entre otros. Pero Juan de Salisbury (1855: 961) adjudicará el uso de este léxico primeramente a Parménides.

Estos nombres (unitas, aequalitas y connexio) son apropiados a las Personas divinas en cuanto a la relativo o relacional en lo divino. Como unitas el Padre es el principio de lo Uno y por tanto principio de la divinidad. El Hijo como aequalitas es la igualdad del Padre, que procede de la misma unitas que es Padre. Y por último, el Espíritu Santo como connexio que procede de ambos es la unión entre los dos, haciendo que los tres sean uno.

Ahora bien, recordemos que Nicolás de Cusa a través de su sistema de la docta ignorancia está más preocupado de la teología negativa. Por lo tanto no se puede nombrar exactamente nada en la Trinidad, porque nombrar es definir, y definir es dividir, cosa que en lo Uno no se puede hacer ya que es absolutamente simple. Es tanto el nivel de abstracción que alcanza el Cusano que según su teología negativa nos advierte que Dios «no es ni Padre, ni Hijo, ni Espíritu Santo, en cuanto que es sólo infinito. Y la infinidad no es, en cuanto infinidad, ni generante, ni engendrada, ni naciente» (Cusa, 1932: 55) y «no se haya en Dios otra cosa que infinidad» (Cusa, 1932: 55-56). Nicolás insistirá que estos nombres se dan «sólo en relación a las criaturas» (Cusa, 1932: 19). 
En el capítulo 7 del libro I observa que en el mundo creado, entre todos los seres existe alteridad, desigualdad y división. Pero estas cosas en sí son finitas, por lo tanto debe haber algo anterior y eterno en lo cual tienen su origen. La unitas es el origen eterno de la alteridad, la aequalitas de la la desigualdad y la connexio de la división. La unitas, aequalitas y connexio son eternas y «de ahí que la unidad, la igualdad y la conexión sean una sola cosa. Y ésta es aquella trina unidad» (Cusa, 1932: 16).

Aunque el Cusano eventualmente seguirá por este camino de la teología afirmativa y la analogia entis, nunca le dará la última palabra. Argumentará que todo lo que podemos afirmar o atribuir a Dios es mero lenguaje convencional; que hacemos trasladación mental de las perfecciones que encontramos en las criaturas hacia Él:

$\mathrm{Y}$ es tan verdad esto acerca de todos los atributos afirmativos, que también el nombre de la Trinidad y de las personas, es decir, del Padre, del Hijo y del Espíritu Santo le son impuestos en relación a las criaturas. Pues como por ser Dios unidad es genitor y Padre, por ser igualdad de unidad es engendrado o Hijo, y por ser conexión de uno y otro es Espíritu Santo... (Cusa, 1932: 50).

En fin, el Cusano nos invita ahora a recorrer un doble camino por medio de la docta ignorancia, el de la teología afirmativa y la teología negativa, en un continuo proceso dialéctico en el conocimiento de Dios y el universo. Todo lo que podemos afirmar de Dios es por medio de la analogía entis, pero ésta siempre quedará corta recordando el principio básico expuesto al comienzo, que entre lo finito y lo infinito no hay proporción. Por último, el hecho de que el gran misterio de la Trinidad se dice en relación a las creaturas lo podemos entender de doble modo: en un sentido desde abajo de que por medio las creaturas y sus relaciones podemos deducir algo sobre las relaciones trinitarias; o también en un sentido desde arriba en que podemos descubrir unas ciertas huellas de la Trinidad en todas las cosas.

\section{E1 Verbo como Aequalitas essendi}

En el capítulo 24 del libro I de De Docta Ignorantia, dedicado a los nombres de Dios y a la teología afirmativa, vemos un esbozo de su teología trinitaria y su relación con la creación. Ahí el Cusano dice que «el engendrar el Padre al Hijo fue crear todas las cosas en el Verbo, y por esto Agustín afirma que el Verbo es arte o idea con relación a las criaturas» (Cusa, 1932: 51). Evidentemente tiene en mente algunos 
pasajes bíblicos como el himno de la Carta a los Colosenses (Col 1,1520) y el prólogo del Evangelio según San Juan (Jn 1,3). Teniendo en cuenta la cristología del Logos que el Cusano está siguiendo por su pensamiento neo-platónico se comprende la importancia específica del Verbo en la creación.

Recopilando algunas ideas hemos visto en las secciones anteriores a Dios como unitas, y que como tal es también entitas. Como entitas en sumo grado es el origen de toda otra entidad. También se dijo que de la unitas procede su aequalitas que es el Hijo. El Hijo como aequalitas unitatis, en el cual se han hecho todas las cosas es también aequalitas essendi:

Dios es la misma entidad de las cosas (rerum entitas), pues es la forma de ser (forma essendi), porque es entidad (entitas). Pero la igualdad de la unidad, es casi igualdad de entidad (entitatis), esto es, igualdad de ser (essendi) o existir (existendi). Ahora bien, hay igualdad de ser (aequalitas essendi) porque en la cosa no hay ni más ni menos, ni nada superior ni inferior (Cusa, 1932: 11).

$\mathrm{Al}$ parecer el Cusano utiliza casi como sinónimos los términos de entitas, esse y existentia. Esto puede ser una influencia de Guillermo de Ockham que contrario a la escolástica no hace distinción entre entitas y esse. También en esto puede tener como referencia a Dietrich de Freiberg que identifica esencia con existencia y es también influencia directa del Maestro Eckhart. Aquí estamos en el difícil terreno de lo máximo en que todas las cosas coinciden. Debemos seguir muy de cerca al Cusano para no perdernos. Anteriormente vimos que la unitas es considerada como entitas. Ahora vemos que la aequalitas unitatis es también aequalitas entitatis que dice es lo mismo que aequalitas essendi o existendi. Siguiendo la lógica, ya que la unitas tiene una aequalitas y esta aequalitas es de la unitas como también del esse, se puede entonces decir que Dios como unitas o entitas es esse. Y ya había dicho que es «la forma de ser». Ahora bien, ¿isignifica esto que Dios es el Ser o quizás el acto de ser o simplemente un ser? En primer lugar debemos advertir que el Cusano estipula que «este nombre de Ser, u otro nombre cualquiera, no es el nombre exacto del Máximo» (Cusa, 1932: 14). Segundo, el Máximo escapa radicalmente al esse de modo que al máximo ser nada se le opone, ni el no-ser ni el ser mínimo (Ibíd.). Tercero, en cuanto al ser de cada cosa dirá que «no es algo en cuanto que es una cosa diversa, sino que su ser procede del máximo ser» (Cusa, 1932: 71). De este modo podríamos entender que en cuanto a la creatura al Cusano no le interesa tanto el tema de la distinción entre esencia y existencia, sino más bien entre quo est (aquello por lo cual algo es) y quod est (aquello que es). Esta distinción versa entre Dios y su creatura, o entre el ser necesario y contingente. Es interesante ver como hace esta 
distinción en su obra De Beryllo de modo que se identifica el quo est con el Padre y el quod est con el Hijo, y al Espíritu como el nexo entre ambos (Flasch, 2003). Si el Hijo da el quod est, o sea «aquello que es», podemos derechamente interpretar el uso de esse como el ser en sí mismo y no sólo como el acto de ser del ente. Así entonces, al decir que el Hijo es Aequalitas essendi lo decimos en su relación a toda cosa considerando su esencia y existencia, tanto en lo divino como en lo creado y así «entonces es claro que el Hijo se llama Hijo porque es igualdad de ser o de entidad, o unidad» (Cusa, 1932: 50-51).

El término Aequalitas essendi tampoco es propio del Cusano. Éste se encuentra ya en las obras de Thierry de Chartres (Hopkins, 1986), de quien Nicolás dirá que es «fácilmente el hombre más inteligente de todos los que ha leído» (Cusa, 1988: 476). En su obra De sex dierum operibus, Thierry de Chartres (2007) dirá que la unitas es el primer ser y la unidad de las cosas, incluso llega a decir que la unitas es el ser de las cosas2. Por ser la unitas el ser de todas las cosas, la igualdad de la unidad debe por tanto también ser la igualdad de ser (aequalitas essendi) de todas las cosas. $\mathrm{Y}$ así como decimos que el Hijo es imagen o concepto del Padre, también se puede decir que es concepto e imagen de todo lo creado. El desarrollo de estas ideas nos llevan a comprender mejor la siguiente cita:

Dios desde la eternidad pudo crear cosas, aun cuando no las creara; con relación a las mismas cosas se dice Hijo, y es Hijo porque es la igualdad de ser de las cosas, por encima o por debajo de la cual las cosas no podrían ser, y así evidentemente es Hijo porque es la igualdad de la entidad de las cosas que Dios podría hacer, aun cuando no hubiera de hacerlas, porque si no pudiera hacerlas no sería ni Dios Padre o Hijo o Espíritu Santo, ni siquiera Dios (Cusa, 1932: 51).

El Hijo es el ser necesario para la existencia de todos los demás seres. El Cusano entiende que todas las cosas existen en y por el Hijo. Se podría quizás mal interpretar este pasaje como modalista en el sentido que dice que Dios «con relación a las mismas cosas se dice Hijo». Pero intenta dejar claro que el Hijo tiene existencia propia independiente y específica que lo diferencia de los demás entes y de las otras Personas

\footnotetext{
2 Es un poco complicado saber lo que Thierry de Chartres quiso decir al afirmar que la unitas es el ser de las cosas. Lo dicho puede ser peligroso en cuanto si Dios y la creatura tienen un mismo ser, entonces, no hay distinción entre los dos, cayendo así en un panteísmo. El Cusano también expresa ese tipo de ideas, y debido a veces a lo abstracto del lenguaje del Cusano, más de alguno, como John Wenck (1988), ha pensado que éste ha querido afirmar que el ser es Dios. Pero ya definimos anteriormente que el Cusano establece la diferencia real entre el ser divino y el ser de la criatura.
} 
divinas. Por otra parte, para ahuyentar ideas panteístas dice que «desde la eternidad pudo crear cosas, aun cuando no las creara» y en cuanto a las cosas «que Dios podría hacer, aun cuando no hubiera de hacerlas», expresa por una parte la omnipotencia de Dios, pero también la libre voluntad divina sobre la creación. A diferencia de algunos pensadores neo-platónicos de origen pagano, la creación no es necesaria para Dios, ni una emanación, sino un acto de su bondad.

Al hablar de la Aequalitas essendi se mezclan conceptos y términos que estábamos acostumbrados a ser referidos a Dios como Trinidad, pero debido a su gran asimilación de la doctrina de la perijoresis el Cusano intercambiará con mucha soltura los conceptos para precisar aún más la grandeza del misterio de la segunda Persona divina como Aequalitas essendi. Al respecto, en el Libro III de De Docta Ignorantia, que se dedica por entero a la cristología, dice que «Dios, que es la igualdad de ser de todas las cosas, es el creador del universo en cuanto que éste está creado para Él mismo» (Cusa, 1932: 128). Por el contexto en que se encuentra la cita sabemos que al hablar de Dios se refiere específicamente al Verbo y no a la Trinidad en general o a alguna de las otras dos Personas. Vemos aquí nuevamente una clara referencia a Colosenses 1,16: «Todo fue creado por Él y para Él».

El Cusano para distinguirse de los platónicos y neo-platónicos, que postulaban la existencia real de las formas o razones de las cosas como ideas en una esfera aparte del mundo físico y distinto de Dios que llamaban el alma del mundo o también mundo de las ideas, afirma que «sólo un ejemplar infinito es suficiente y necesario» (Cusa, 1932: 94). Este ejemplar es obviamente el Verbo, «en el cual todas las cosas están, como las cosas ordenadas en el orden, y el cual complica todas y cada una de las distintas razones de las cosas de modo adecuadísimo» (Ibíd.).

Ahora es un buen momento para hablar de un par de conceptos que se acompañan como contrarios y complementos que son centrales en el pensamiento del Cusano. Se trata del binomio de complicatio y explicatio. La complicatio se refiere a al hecho de unir, compilar cosas diversas entre sí en uno solo. En este sentido todas las razones de las cosas están complicadas en lo absoluto o el Ejemplar infinito. Y la explicatio es el traspaso de la idea de la cosa en la mente divina, es decir desde lo absoluto hacia su concretización en el mundo creado. A este ente concreto el Cusano lo llama contracto. En esta dinámica de la complicatio y explicatio veremos más adelante al Verbo encarnado como el lugar de esta complicación y explicación, es la bisagra entre los dos infinitos: el infinito absoluto de Dios y el infinito privativo de la creación. La especulación metafísica de la dinámica de esta interrelación es el punto central de su pensamiento (Brient, 2002). 


\section{Relación Trinidad y creación}

Aún no hemos respondido a la pregunta de cómo es posible que a pesar de la necesidad de un único ejemplar las cosas existan de modo tan diverso. Respecto a esto el Cusano dice que «viendo las diversidades de las cosas, nos admiramos de cómo una única razón simplicísima de todas las cosas sea diversa en cada una de ellas» (Cusa, 1932: 95). A través de esta cita queremos hacer mención a la solución que encuentra a este problema por medio de su docta ignorancia. Veremos ahora brevemente como articula su teoría del conocimiento que se divide en tres esferas: sentidos, razón e intelecto. Este esquema se encuentra también en otros autores como San Agustín, pero el Cusano lo adapta y explaya a su modo.

El primer paso del conocimiento es a través de los sentidos que captan a las cosas en su diversidad y alteridad. La noción de la alteridad y diversidad se percibe primeramente por los sentidos. La razón luego ordena y clasifica todo rigiéndose por ciertos principios de la lógica. Pero a pesar del conocimiento claro y preciso que nos entrega el razonamiento humano el Cusano nos invita a superar la lógica humana limitada por las conjeturas. Apela a una suerte de intuición superior que nos permite adentrarnos más en el misterio del ser. Al respecto dice que «nos admiramos» al descubrir una misma ratio presente en cada cosa. Esta ratio puede considerarse también como la quididad de las cosas que todos los filósofos han buscado desde siempre. Según el pensamiento del Cusano esta especie de intuición superior es lo que intelecto, que es la esfera más alta del conocimiento humano. Este intelecto está muy relacionado con lo que llamamos contemplación y es la capacidad de captar la verdad más profunda de cada cosa. Es una cierta capacidad de abstracción que permite, a pesar de las diversas razones o formas, sobrepasar el principio de no-contradicción y así captar que «la igualdad máxima es la razón de todas las cosas» (Cusa, 1932: 34). Esta «igualdad máxima» es el mismo Verbo y como tal «no es distinta o diferente de ninguna cosa» (Cusa, 1932: 10). Por tanto, la razón de los seres es distinta entre ellos pero no en relación a Dios, «y cuando se dice que Dios creó al hombre por otra razón que a la piedra, esto es verdadero con relación a las cosas, no al Creador» (Cusa, 1932: 95).

En este sentido el Verbo es no-otro (non-aliud), concepto que el Cusano utilizará mucho más adelante en su vida para hablar de Dios. El máximo, como máximo coincide necesariamente con lo mínimo y así abarca todo y es a la vez mayor e inferior a todas las cosas. En la igualdad máxima que es razón de todas las cosas se da simultáneamente todo lo que es, lo que podría ser, lo que no es, incluso lo contradictorio entre sí. En Él 
todas las cosas son Él mismo, de modo que en Él es lo mismo ser y noser.

Veamos momentáneamente la cuestión del no-ser en el Máximo. Habíamos establecido ya que todos los entes participan de la entidad (entitas) que es Dios y en este sentido Él es la esencia del ser ente de cada ente. Por una parte, si quitamos a Dios de la criatura no quedaría sino la nada (Cusa, 1932: 71). Pero por otra parte, nosotros no conocemos a la entitas en sí misma, sino sólo por medio de la participación que se da de ella en los entes. Si por medio de una abstracción mental nos olvidamos de la participación de los entes en la entitas y nos enfocamos solamente en la entitas misma al parecer nos quedamos con nada, pues qué es la entitas sin entes sino nada (Cusa, 1932: 35). En esta misma línea el Cusano cita a Dionisio diciendo que «el concepto de Dios más se aproxima a la nada que a algo» (Ibíd.). Descubriendo un doble sentido en el Cusano (cosa que es muy frecuente en sus escritos), podemos atrevernos a decir que «el concepto de Dios» al que se refiere no es sólo la apreciación de Dios que tenemos en nuestra mente sino también el mismo Verbo eterno que es el concepto que Dios tiene de sí mismo. Ahora bien, sabiendo que sólo «hallamos a Dios por remonición de la participación de los entes» (Ibíd.) nos encontramos ahora con una gran contradicción. Pues la única forma de conocer a Dios es abstrayéndose de los entes, pero esto nos lleva visualizar nada, por tanto qué es lo que podemos conocer sino nada. Esta es la apuesta de Nicolás de Cusa, que por medio de la docta ignorancia comprendemos incomprensiblemente que «Dios está en la nada» (Cusa, 1932: 71).

Siguiendo el mismo tema pregunta: «¿cómo se entiende que la pluralidad de las cosas exista, porque Dios esté en la nada, no teniendo la nada ninguna entidad?» (Cusa, 1932: 72). ¿Desde dónde entonces proviene el universo según el Cusano? Sabemos ya que Dios «hizo en el Verbo uno todas y cada una de las cosas» (Cusa, 1932: 95) pues el Padre al engendrar al Hijo hizo todas las cosas en Él. Si en Dios todas las cosas coinciden, el ser, estar o hacer desde la nada se conjugan. Podemos así entonces atrevernos a pensar aquí en un movimiento kenótico del Verbo en relación a la creación en que se hace a un lado para dar espacio y tiempo para dejar ser a todos los demás seres. De modo que el Verbo, el concepto de Dios, en el cual se realiza la creación no solo está en la nada sino que es la nada desde la cual nace la creación entera. En la creatio ex nibilo podemos suponer que decir que Dios crea de la nada es lo mismo que decir que crea desde sí mismo.

Ahora veremos los modos trinos del ser o las buellas que hay de la Trinidad en todos los seres creados. En resumen se trata de cómo la dinámica trinitaria se refleja en todo ente, o de otro modo, de cómo cada 
ente nos revela algo sobre como es la Trinidad. Veremos en la siguiente cita un buen resumen de esta teoría del ser del Cusano:

De ahí que Dios sea Padre, porque engendró la igualdad de la unidad, y también Espíritu Santo, porque es el amor de uno y otro, y todas estas cosas con relación a las criaturas. Pues la criatura toma el ser porque Dios es Padre, se perfecciona porque es Hijo, y concuerda con el orden universal de las cosas porque es Espíritu Santo. Y éstas son las huellas que hay en cualquier cosa de la Trinidad. Y ésta es la sentencia de Aurelio Agustín, exponiendo este pasaje del Génesis: 'En principio Dios creó el cielo y la tierra', diciendo que Dios, porque es Padre, había creado los principios de las cosas (Cusa, 1932: 51).

Se trata de concebir a la Trinidad en relación al ser creado como causa eficiente, formal y final de todas las cosas (Cusa, 1932: 95). Esta idea no sólo se ve en el Cusano sino también anterior a él en autores como Alberto Magno y Thierry De Chartres.

El Padre se puede considerar como causa eficiente «pues la criatura toma el ser porque Dios es Padre». O sea, se le atribuye al Padre el dar el acto de ser, o también como habíamos mencionado antes el quo est. Se dice por otra parte que la criatura «se perfecciona» por medio del Hijo. En su pensamiento la perfección se da en la medida que algo es, y es menos perfecta en cuanto no-es. Ahora bien, el ser de las creaturas se define tanto por lo que son en sí mismas como por su no-ser en relación a lo demás. Del Hijo las creaturas reciben su modo de ser, o forma, el quod est, y por esto se perfeccionan por Él, pues su perfección consiste en ser lo que son y no otra cosa, y esto lo reciben por medio de la Aequalitas essendi. En cuanto a que las creaturas «concuerdan con el orden universal de las cosas» por medio del Espíritu Santo, esto se debe a que Éste es causa final de todas las cosas. Es pues con relación al fin que las cosas se ordenan entre sí en su relación con todo el universo. También se puede decir que algo es causa final porque ésta da la razón por la cual algo se causó en primer lugar, responde al para qué de una cosa. Y así como el Espíritu es «el amor de uno a otro» en la Trinidad, en relación a las creaturas también es el amor, y por tanto es la razón última por la cual han llegado a existir. Así, si decimos que el Padre creó todo ex nibilo, y esto lo habíamos entendido como la creación desde el Hijo, podemos decir también que el Padre creó todo ex amore, y esto se entiende que creó desde el Espíritu Santo.

En relación al misterio de la creación Nicolás recuerda el pasaje del Génesis: In principio creavit Deus caelum et terram (Gn 1,1). Lo interpreta, siguiendo a Agustín (1845b) y en general a toda la corriente cristiano- 
platónica, de modo metafísico. "In prinicipio» no se trata de un inicio temporal de la creación sino el principio ontológico en el cual todo el universo existe. En esta interpretación el Hijo es el Principio en que el Padre creó todas las cosas. Agustín (1845c) enseña además que el Padre es principio sin principio, que es principio de la deidad, que el Hijo es principio de principio, y de cómo no son dos ni tres principios sino uno solo. Nicolás, resumiendo a Agustín, dice que «Dios, porque es Padre, había creado los principios de las cosas». Declara que el Padre creó «los principios de las cosas», pero según Agustín (y la toda la doctrina cristiana) a quien el Cusano está siguiendo, hay sólo un principio de las cosas, el cual no es creado. Según el contexto trinitario pareciera ser que Nicolás se refiere al Hijo y al Espíritu Santo como estos "principios» y al decir que son creados quiso decir que proceden del Padre por ser éste principio de la deidad como lo enseña Agustín3.

Al hablar de los principios de las cosas en relación a la Trinidad el Cusano nos está introduciendo a su idea de las «huellas que hay en cualquier cosa de la Trinidad» o también de los modos trinos del ser. Todos los seres creados están compuestos por tres principios, a imagen de la Trinidad, que permiten su existencia. En las estructuras clásicas del ente, existencia y esencia, materia y forma, potencia y acto, y con la respectiva unión entre estos principios, el Cusano ve las huellas de la Trinidad.

Estas huellas trinitarias las explica diciendo que Dios como máximo absoluto es la medida de todas las cosas. Y en la misma línea dice que en las cosas «la unidad del ser es trinidad» (Cusa, 1932: 41). Todo ser como uno tiene una estructura trinitaria. Según una primera apreciación el Cusano adopta, como otras veces lo hemos mencionado, la analogia entis. Por medio de las creaturas se puede llegar al Creador que es la medida de todas las cosas, y sin las creaturas «no entenderíamos que él mismo pudiera ser medida de todas las cosas» (Ibíd.). Nos recuerda que «al máximo, aunque esté infinitamente por encima de toda trinidad, le llamamos trino» (Ibíd.). Manteniendo por un lado el principio de desproporcionalidad incursionamos en el frágil camino de la analogía, que es el único camino que nos queda. Por eso «tanto el nombre como el concepto nuestro de trinidad... nunca conviene al máximo, sino que se aparta infinitamente de aquella máxima e incomprensible verdad» (Ibíd.). Así, aunque descubrimos una cierta trinidad en las cosas creadas, y esto es una cierta imagen de Dios, este último supera cualquier analogía que le podríamos aplicar, pues llamamos trino al Máximo en relación a las creaturas y no en sí mismo. Pero aún así el Máximo es medida de todas

3 En otra ocasión Nicolás de Cusa (1932: 98) habla del Espíritu como creado para decir que procede. 
las cosas contractas pues ellas están en Él como prototipos de modo complicado.

Aunque todos los seres subsisten trinitariamente cada ser es único y diferente en relación a todos los demás, pues la «unidad de ser consiste en la pluralidad» (Cusa, 1932: 42). La causa de la diversidad de los entes se debe a la diversidad de formas, lo cual se traduce en la diversidad de géneros y especies. Ahora bien, la diversidad de formas se debe a que en Dios no puede haber otra cosa igual a Dios que no sea Dios mismo, de modo que toda la creación participa de algún modo de la infinitud y perfección de Dios. Esta participación de los entes tampoco puede repetirse exactamente entre ellos en el sentido de que un ente no puede ser otro. Tienen ciertas similitudes pero ninguna es totalmente igual a otra. Y así como todos participan de modo diverso de la perfección divina, aunque algunos son más perfectos que otros, ninguno puede ser máximamente perfecto ya que tendría que ser infinito para lograr esto y la infinitud en sí no puede estar contracta.

Estos puntos serán importantes tener en cuenta para cuando hablemos sobre la encarnación del Verbo, pues al parecer ningún contracto puede ser máximo, y por tanto la encarnación una imposibilidad. Hemos visto además el rol del Verbo como Aequalitas en su relación con los entes creados. Anteriormente habíamos reflexionado sobre la percepción de la Trinidad que tiene el Cusano. Descubrimos su profunda noción de la perijorésis en el concepto de la Unitas. A través de este concepto clave explicará a Dios y a la creación entera. La unitas es el concepto más básico que se encuentra en la raíz de todo. Como concepto trascendental todos los seres participan de él, los cuales también tienen una estructura trina. Es sólo por medio de la igualdad de la unidad, la Aequalitas unitatis, que es también la Aequalitas essendi que podemos descubrir algo de la Unitas absoluta como también de la unitas de cada ser. En relación a nosotros la Aequalitas es el medio para trascender a Dios, en primer lugar por medio de la inmanencia de lo divino en la creación; en segundo lugar, por lo que veremos a continuación, porque la Aequalitas se hizo carne.

\section{Encarnación como la contracción máxima de la Aequalitas essendi}

Anteriormente hemos visto el rol de la Aequalitas en la creación, pero por el hecho de que ahora tocaremos el tema de la encarnación no debe hacernos pensar que podemos olvidar lo ya dicho o pensar que estamos cambiando ahora a otro tema. En su lógica de la coincidencia de los 
opuestos la encarnación juega parte esencial en la creación. Ante la clásica pregunta de si el Verbo se hubiese encarnado aunque no hubiese habido pecado original, el Cusano habría respondido afirmativamente (McGinn, 2002).

El planteamiento que hace el Cusano de la encarnación parte como una pregunta filosófica. Después de haber indagado sobre el Máximo absoluto que es Dios y el máximo contracto que es el universo en los libros I y II de su De Docta Ignorantia se pregunta en el Libro III si acaso un contracto puede ser en sí mismo máximo. Llega a la conclusión de que la única manera que un contracto pudiera ser máximamente, es que tendría que ser Dios y criatura a la vez. Esta criatura complicaría en sí misma todas las razones y perfecciones de su especie, y podría existir sólo si subsistiera en Dios. Ahora bien, esta criatura, para ser realmente máxima no podría asumir cualquier naturaleza. Tendría que asumir una naturaleza que fuera a la vez apropiada para el Máximo, de modo que a través de esta criatura podría ser la razón, forma y perfección de todas las cosas existentes. La única naturaleza creada que podría cumplir con este requerimiento sería la naturaleza humana. Como naturaleza media, complica en sí misma las naturalezas de todos los seres creados, tanto los espirituales como los ángeles como también todos los demás seres materiales. El Cusano adopta el antiguo concepto griego de microcosmos para hablar del ser humano. De este modo, el Máximo al asumir una naturaleza humana concreta, complica máximamente en esta misma naturaleza humana todas las cosas. Esta unión se podría dar sólo en la hipóstasis del Hijo ya que como Aequalitas essendi complica de por sí todas las cosas en su Persona, y así al asumir una naturaleza humana puede perfectamente a través de esta misma naturaleza seguir siendo la razón, forma e igualdad de todo ser.

El Cusano finalmente concluye con su caso hipotético acerca de la encarnación para manifestar no sólo la posibilidad sino por sobretodo su realización en Jesucristo. Dejemos que el mismo cardenal nos resuma lo hasta ahora dicho:

Pero Dios, que es la igualdad de ser de todas las cosas (aequalitas essendi omnia), es el creador del universo en cuanto que éste está creado para Él mismo. Así, pues, la igualdad suma y máxima de ser todas las cosas absolutamente (aequalitas summa atque maxima essendi omnia absolute) es aquella a la cual la propia naturaleza de la humanidad se uniría, como el mismo Dios, asumiendo la humanidad, sería todas las cosas contractas en la misma humanidad, del mismo modo que es absolutamente la igualdad de ser de todas las cosas (aequalitas essendi omnia). Aquel hombre, pues, que subsistiera por unión con la máxima igualdad de ser (aequalitate essendi), sería 
hijo de Dios, como el Verbo, en el cual todas las cosas están hechas; o sería la misma igualdad del ser (essendi aequalitas), que se llama hijo de Dios, según se ha manifestado al principio; y no dejaría de ser hijo del hombre, como no dejaría de ser hombre, según se dirá más abajo (Cusa, 1932: 128).

En esta pura cita se utiliza cinco veces la terminología de aequalitas essendi. El concepto de aequalitas essendi en relación a la unión hipostática se basa en la persona divina del Verbo, lo cual no quita que no esté relacionado con la naturaleza humana asumida. El Cusano se preocupa de contemplar la Aequalitas essendi desde ambas perspectivas, tanto la divina como la humana. Es muy cuidadoso de no mezclar ni confundir las naturalezas pero tampoco cae en el error de excluirlas entre sí, ni tampoco menospreciar una naturaleza en favor de la otra. Encuentra ese perfecto equilibrio que permite complementar ambas naturalezas. Ya hemos visto como el Verbo por su condición divina es Aequalitas essendi, ahora interesa seguir resaltando como incorporar a este concepto la naturaleza humana que ha asumido.

En esta línea el Cusano interpretará la concepción de Cristo como Primogénito de toda la creación (cfr. Col 1,15-20). Argumentará que la encarnación no es sólo algo hipotético, ni tan tampoco sólo una realidad soteriológica, sino que fue necesaria para la misma creación. La encarnación no sólo estaba en la intención original de Dios al crear el mundo, sino que fue también el modelo, arquetipo del universo y por esto con razón se le puede llamar Primogénito. El Verbo en su pura divinidad no puede ser considerado Primogénito ya que este título supone una realidad creada. Pero sí se logra comprender al considerar al Verbo como encarnado aún antes que esto ocurriese en el tiempo (Cusa, 1932: 129). Pues en Dios no puede haber un antes y un después, un momento que en el Verbo no estuviese encarnado y un momento en que sí lo estuviese, esto sería una mutación lo cual es imposible en Dios. De modo que la idea de la encarnación y la creación han coexistido siempre en el Verbo y por esto que el Cusano decía que el «engendrar el Padre al Hijo fue crear todas las cosas en el Verbo» (Cusa, 1932: 51).

Veamos un poco más que entiende el Cusano por el hecho de que el Primogénito es ratio a través de su humanidad. Declara que todas las cosas salen de la humanidad de Cristo «para que aquello que son puedan serlo en un orden y modo mejor» (Cusa, 1932: 129). Y también dice que «toda creatura existe en la misma humanidad suma y perfectísima que complica universalmente todo lo creable, de modo que toda plenitud tenga su morada en Él» (Cusa, 1932: 131). Postula además que «Cristo es la razón de todas las razones que se ha encarnado, porque el Verbo se hizo carne. Por lo tanto, Jesús es el fin de todo» (Cusa, 1932: 154). En 
Cristo existe un doble movimiento con respecto a la creación. Todo sale de Cristo por principio de emanación y todo vuelve a Él como a su fin por principio de reducción (Cusa, 1932: 127).

En Dies Santificatus, su sermón de Navidad del año 1440, el mismo año en que escribió De Docta Ignorantia, tenemos quizás una de las mejores explicitaciones que el Cusano hace acerca de este tema cristológico. Allí llega a afirmar que «si Dios no hubiera asumido la naturaleza humana, ya que ella siendo en sí misma como algo intermedio complica todo lo demás, el universo no sería perfecto, ni siquiera existiría (Cusa, 2003: 368). La encarnación, según el Cusano, es absolutamente necesaria para la creación del universo. Sin encarnación no hay creación. Como antes se mencionó, Dios no podía asumir cualquier naturaleza creada sino sólo una que resume en sí a todas las demás, o como diría el mismo Cusano, que complica todas las demás cosas. La naturaleza humana es aquella naturaleza media que en sí misma contiene todas las demás naturalezas, es como se mencionó anteriormente un microcosmos. Ahora vemos que la naturaleza humana no sólo era apta para recibir a Dios, sino que necesaria para que a través de ella Dios creara todas las demás cosas. Es en esta contracción del Verbo en la humanidad concreta de Jesucristo que se encuentra la razón de toda otra contracción y de su ordenamiento en el universo.

Por lo cual, todas las cosas que son existen como contractas por aquel que es absolutamente, y ellas mismas, en cuanto que son contractas, son por aquel al cual la contracción está sumamente unida. Y así como Dios sea creador en primer lugar, y en segundo Dios y hombre, una vez creada la humanidad, asumida supremamente en la unidad de Él, casi sea la contracción universal de todas las cosas, unida hipostáticamente y personalmente a la igualdad de ser de todas las cosas (aequalitati omnia essendi), por Dios absolutísimo, mediante la contracción universal que es la humanidad (Cusa, 1932: 128-129).

Aquí el Cusano ha declarado expresamente que la encarnación ha sido necesaria para la creación. Comienza diciendo que todas las cosas contractas existen gracias al ser absoluto, es decir Dios. Pero en seguida agrega que estas son contractas "por aquel al cual la contracción está sumamente unida». Sabemos que al que se refiere es al Verbo pero el hecho de destacar su unión con una naturaleza humana es un claro indicio de que es en relación a la unión hipostática que las cosas contractas pueden ser contractas. De modo muy resumido podemos recordar y aplicar aquí lo dicho anteriormente de la distinción entre el quo est y el quod est. El Padre como absoluto y potencia máxima se relaciona 
con el quo est, o sea el que da el ser, mientras que el Hijo, pero más específicamente mediante su naturaleza humana, es decir por su contracción, se relaciona con el quod est, es decir da forma de ser.

Sólo a través del misterio de la encarnación (recordando también que este misterio no se debe considerar en el tiempo sino en la intención) pudo Dios crear todo lo contracto y seguir relacionándose con ello. Sin la encarnación, donde Dios es todas las cosas contractamente, el universo como lo conocemos existiría sólo potencialmente en Dios, de modo abstracto y absoluto. Siguiendo la lógica de la encarnación, Nicolás de Cusa enseña que el universo es llevado desde la potencia al acto por el Espíritu Santo (Cusa, 1932: 133). Lo que finalmente lleva al acto es lo que se encuentra complicadamente en la máxima contracción del Verbo. Y por esto el universo es lo que es y no de otro modo, pues tiene su ejemplar en el microcosmos que subsiste antes del tiempo en la hipóstasis del Verbo.

Al encarnarse la Aequalitas essendi es considerada como la «contracción universal de todas las cosas». En otra ocasión el Cusano dice que el Verbo encarnado es «entidad universal contracta» (Cusa, 1932: 127). Anteriormente habíamos dicho que Dios es entidad o entitas, y siguiendo la teoría de la analogia entis, es la entidad de las cosas o forma de ser de todas las cosas. Como Aequalitas el Verbo es la entidad universal, pero por la encarnación lo es no sólo absolutamente sino también contractamente, es decir en un ser concreto y material. Resumiendo, podemos decir que Cristo es como el trascendental de todo el universo, es el mismo principio, centro y fin que traspasa todo.

\section{Conclusión}

Sin duda, aún queda mucho por decir de la cristología de la Aequalitas en el Cusano. En todo caso, y resumiendo, podemos decir que el concepto cristológico de Aequalitas ayuda a ampliar la noción de Cristo, del universo, del cristianismo y de la vida del cristiano en sí misma.

Lamentablemente estaba fuera del alcance de este artículo poder entrar en la relación entre antropología y cristología la cual es notable en el pensamiento del Cusano. Esta relación se basa principalmente en que por ser Aequalitas, Cristo es encontrado en la misma humanidad de cada ser humano, y más específicamente en el estadio del intelecto donde actúa más específicamente la gracia. Al entender a Cristo como Aequalitas essendi tenemos no sólo una llave que nos permite reinterpretar toda la realidad a la luz del misterio de Cristo, sino que también a través del 
mundo creado pero sobre todo a través del hombre descubrimos el misterio de Dios. En su pensamiento se observa un giro antropocéntrico anticipando así la época del Renacimiento.

A un nivel cognoscitivo, según el principio de no-proporcionalidad entre lo finito y lo infinito, no es posible la analogia entis con respecto a Dios, pero en Cristo el hombre tiene un acceso intelectual a Dios y toda la realidad. El Cusano nos ha hecho ver que a pesar de que mientras estamos en esta vida no hay ningún conocimiento que puede ser perfecto, sobre todo cuando se trata de algo tan abstracto como la cuestión del ser, se puede llegar a ciertas conjeturas o aproximaciones que nos permiten acercarnos al objeto final de nuestra intelección que es Dios mismo. Su método de la docta ignorancia nos ayuda a buscar la verdad de modo dialéctico, sobre todo cuando intentamos aprehender a Dios mismo, causa de todo ser. Este método epistemológico nos hace ser mucho más humildes pero también mucho más audaces en nuestra búsqueda de la Verdad. Para el mismo Cusano esto significó pensar en cosas que estaban adelantadas a su época, como por ejemplo sugerir una reforma eclesial, postular antes de Copérnico que la tierra no está en el centro del universo, que puede existir vida en otros planetas, encontrar puntos en común con el Islam, entre muchas otras. El cardenal también recurre a ejemplos matemáticos que podríamos asociar a la teoría de los conjuntos (Mcfarlane, 2004), como también algunos ejemplos sobre la coincidencia entre lo mínimo y máximo que se podrían comparar con la física actual donde se postula que la oscuridad no existe sino lo que existe es la luz mínima o ausencia de esta misma.

Hablando a un nivel más ontológico, Cristo es visto como el trascendental que traspasa toda realidad. Tenemos en el Cusano un apoyo para una teología tanto descendente como ascendente. Cristo como la encarnación de la Igualdad de todo ser es el único mediador entre el mundo creado y su Creador. De este modo podemos decir que ningún ser escapa a Cristo, sobre todo cuando se trata de la realidad humana. Este modo de comprender a Cristo ayuda a descubrirlo donde uno no espera encontrarlo. Así, el pensamiento del Cusano nos ayuda hoy en día a no limitar a Dios ni a su voluntad entendiendo que en Él son infinitas las posibilidades de los seres y que todas éstas se dan simultáneamente en Él sin oposición ni contradicción.

$\mathrm{La}$ ciencia, igualmente es un lugar para encontrar a Cristo. Esto lo demostraron dos astrónomos jesuitas, George Coyne y Guy Consolmagno, en el programa radial estadounidense «On Being» el día 10 de marzo del 2011. En la entrevista los jesuitas hicieron algunas menciones explícitas a Nicolás de Cusa, pero también se nota en el transfondo de sus apreciaciones sobre la ciencia, el universo y el 
conocimiento de Dios la influencia del Cusano o por lo menos implicaciones derivadas de su pensamiento.

Por otra parte se puede establecer una base teológica para el diálogo sobre cuestiones éticas. David J. De Leonardis (1998) da un claro ejemplo de esto en su obra Ethical Implicationes of Unity and the Divine in Nicholas of Cusa.

Las posibilidades del diálogo ecuménico e interreligioso se amplían enormemente. Aplicando su método se puede encontrar a Cristo en todas las religiones y en toda búsqueda honesta de la Verdad.

La cristología del Cusano ayuda a apreciar todo el conocimiento antropológico, como también a valorar la singularidad de cada humanidad contracta, es decir, de cada persona humana en particular, incluso en el más miserable. Desde esta perspectiva, su interpretación de la igualdad de Cristo con cada ser humano hasta el más «mínimo» de ellos, como también su concepción del pecado como un no-ser de la propia identidad humana, puede constituir un gran respaldo para la dimensión pastoral.

Desde un punto de vista eclesiológico se puede fundamentar la constante renovación de la Iglesia, ya que como la esencia de ésta es la unión con Cristo y Éste es infinito, por lo tanto también pueden ser variadas las formas que adopta la Iglesia para conformarse más con Cristo.

Por otra parte, este tipo de pensamiento podría ser también una fascinante alternativa para aquellos que se sienten atraídos hoy en día por corrientes de gnosticismo y eclecticismo, pues aparte de ser un pensamiento integral, por sus raíces neo-platónicas es también la base para una vida mística relacionada con un conocimiento más profundo del universo.

En Cristo, en quien habita la plenitud de la divinidad corporalmente (Col 2,9) descubrimos la clave de interpretación a todas las cuestiones más profundas del ser humano. El paradigma de la Igualdad del ser encarnada nos enseña que nada puede ser indiferente para el creyente y éste aprende por la docta ignorancia que debe relativizarlo todo para llegar a lo Absoluto: «examínenlo todo y quédense con lo bueno» (1 Tes $5,21)$.

\section{REFERENCIAS}

-Agustín, San (1845a). De Doctrina Christiana libri quatour. En J. P. Migne (Ed.), Patrologia Latina (Vol. 34, págs. 15-122). París: Migne. 
-Agustín, San (1845b). De Genesi ad Litteram Imperfectud. En J. P. Migne (Ed.), Patrologia Latina (Vol. 34, págs. 219-246). París: Migne.

-Agustín, San (1845c). De Trinitate. En J. P. Migne (Ed.), Patrologia Latina (Vol. 42, págs. 354-430). París: Migne.

-Albertson, D. (2006). That He might fill all things: Creation and Christology in two treatises by Nicholas of Cusa. International Journal of Systematic Theology, 8 (2), 184-205.

-Balthasar, H. U. von (1997). Teológica (L. Piossek \& J. P. Tosasus, Trads., Vol. II). Madrid: Encuentro.

-Balthasar, H. U. von (1998). El Nudo, Nicolás de Cusa. En H. U. von Balthasar, Gloria. Una Estética Teológica (E. Suara, Trad., Vol. V, págs. 195232). Madrid: Encuentro.

-Brient, E. (2002). Meister Eckhart and Nicholas of Cusa on the "Where" of God. En C. Bellito \& T. Izbicki (Eds.), Nicholás of Cusa and His Age: Intellect and Spirituality (págs. 127-150). Leiden: Brill Academic Publishers.

-Chartres, T. de (2007). Traité de six tours de la creation. (D. Kmiecik, Trad.) Recuperado el 16 de Marzo de 2012, de: http://users.skynet.be/etc /Art_Drivers /Tsjctc.html.

-Cordovilla, A. (2004). Gramática de la Encarnación. Madrid: Universidad Pontificia Comillas.

-Cusa, N. de (1988). Apologia Doctae Ignorantiae. En J. Hopkins (Ed.), Nicholas of Cusa's debate with John Wenck (págs. 459-492). Minneapolis: A. J. Banning Press.

-Cusa, N. de (1932). De Docta Ignorantia. En E. Hoffman \& R. Klibansky (Eds.), Opera Omnia (Vol. I). Lipsiae: Academiae Litterarum Heidelbergensis.

-Cusa, N. de (1984). La Docta Ignorancia. (M. Fuentes Benot, Trad.) Buenos Aires: Orbis.

-Cusa, N. de (2003). Sermo XXII: Dies Sanctificatus. En J. Hopkins (Ed.), Nicholas of Cusa's Early Sermons: 1430-1441 (págs. 358-376). Loveland: A. J. Banning Press.

-De Leonardis (1998). Ethical Implicationes of Unity and the Divine in Nicholas of Cusa. Washington D.C.: The Council for Research in Values and Philosophy.

-Euler, W. A. (2000). Does Nicholas of Cusa Have a Theology of the Cross? The Journal of Religion, 80 (3), 405-420.

-Flasch, K. (2003). Nicolás de Cusa. (C. Ruíz-Garrido, Trad.) Barcelona: Herder.

-Haubst, R. (1956). Der Christologie Des Nikolaus Von Kues. Freiburg: Herder.

-Heidegger, M. (2001). Introducción a la Metafísica (A. Ackermann Pilári, Trad.) Barcelona: Gedisa.

-Hubert, A. (2009). Balthasar y el Nudo de la Metafísica de Nicolás de Cusa. Teología y Vida 50 (1-2), 421-432.

-Hopkins, J. (1986). A Concise Introduction to the Philosophy of Nicholas of Cusa. Minneapolis: A. J. Banning Press. 
-Mcfarlane, T. J. (2004). Nicholas of Cusa and the Infinite. Recuperado el 18 de Marzo de 2012, de Integral Science: http://integralscience.org/cusa.html.

-McGinn, B. (2002). Maximum Contractum et Absoultum: The Motive for the Incarnation in Nicholas of Cusa and his Predecessors. En C. Bellito \& T. Izbicki (Eds.), Nicholás of Cusa and His Age: Intellect and Spirituality (págs. 151175). Leiden: Brill Academic Publishers.

-Salisbury, Juan de (1855). De Septem Septenis. En J. P. Minge (Ed.), Patrologia Latina (págs. 945-964). París: Migne.

-Tilliette, X. (1993). Le Christ de Philosophes. Namur: Culture et Vérité.

-Wenck, J. (1988). De Ignota Litteratura. En J. Hopkins (Ed.), Nicholas of Cusa's debate with John Wenck (págs. 425-456). Minneapolis: A. J. Banning Press.

Sumario: Introducción: el Cusano y el problema del ser; 1. Algunos conceptos claves del Cusano en De Docta Ignorantia; 2. La Trinidad como Unitas, Aequalitas y Connexio; 3. El Verbo como Aequalitas essendi; 4. Relación Trinidad y creación; 5. Encarnación como la contracción máxima de la Aequalitas essendi; Conclusión; Referencias. 